Pacific Journal of Mathematic 


\section{CHARACTERIZATIONS OF CERTAIN MAPS OF CONTRACTIVE TYPE}

\section{ChI Song Wong}

The following result is obtained. Let $f$ be a self map on a nonempty complete metric space $(X, d)$. Then the following conditions are equivalent: (i) For any $\epsilon>0$, there exists $\delta(\epsilon)>0$ such that $d(f(x), f(y))<\epsilon$ whenever $\epsilon \leqq d(x, y)<\epsilon+\delta(\epsilon)$. (ii) There exists a function $w$ of $[0, \infty)$ into $[0, \infty)$ such that $w(s)>s$ for all $s>0, w$ is lower semicontinuous from the right on $(0, \infty)$ and $w(d(f(x), f(y))) \leqq d(x, y), x, y \in X$.

1. Introduction. In 1969, E. Keeler and A. Meir [3] obtained the following result.

Theorem A. (Keeler and Meir). Let $f$ be a self map on $a$ nonempty complete metric space $(X, d)$. Suppose that for any $\epsilon>0$, there exists $\delta(\epsilon)>0$ such that $d(f(x), f(y))<\epsilon$ whenever $\epsilon \leqq d(x, y)<$ $\epsilon+\delta(\epsilon)$. Then $f$ has a unique fixed point $x_{0}$ and $\left\{f^{n}(x)\right\}$ converges to $x_{0}$ for all $x$ in $X$.

Theorem A generalized the following result of D. W. Boyd and J. S. W. Wong [1] (and therefore, an earlier result of E. Rakotch [4]).

Theorem B. (Boyd and Wong). Let $f$ be a self map on a nonempty complete metric space $(X, d)$. Suppose that there exists a self map $\Phi$ on $[0, \infty)$ such that $\Phi$ is upper semicontinuous from the right, $\Phi(t)<t$ for $t>0$ and $f$ is $\Phi$-contractive :

$$
d(f(x), f(y)) \leqq \Phi(d(x, y)), \quad x, y \in X
$$

Then $f$ has a unique fixed point $x_{0}$ and $\left\{f^{n}(x)\right\}$ converges to $x_{0}$ for all $x$ in $X$.

In this paper, equivalent conditions in terms of monotone transformations are obtained. These will show that the essential difference between Theorems A and B is a matter of imposing monotone transformations on the left side or right side of certain inequalities.

\section{Main results.}

THEOREM 1. Let $f$ be a self map on a nonempty complete metric space $(X, d)$. Then the following conditions are equivalent: 
(i) For any $\epsilon>0$, there exists $\delta(\epsilon)>0$ such that $d(f(x), f(y))<\epsilon$ whenever $\epsilon \leqq d(x, y)<\epsilon+\delta(\epsilon)$.

(ii) There exists a self map $w$ of $[0, \infty)$ into $[0, \infty]$ such that $w(s)>s$ for all $s>0, w$ is lower semicontinuous from the right on $(0, \infty)$ and

$$
w(d(f(x), f(y))) \leqq d(x, y), \quad x, y \in X .
$$

Proof. (i) $\Rightarrow$ (ii). Let $\epsilon>0$. (i) implies that $f$ is contractive: $d(f(x), f(y))<d(x, y)$ for distinct $x, y$ in $X$. So

$$
d(f(x), f(y))<\epsilon \text { whenever } d(x, y)<\delta(\epsilon) .
$$

Define $w(0)=0$ and

$$
w(\epsilon)=\sup \{\delta(\epsilon)>0: \delta(\epsilon) \text { satisfies }(*)\} .
$$

Then $w$ is an increasing function of $[0, \infty)$ into $[0, \infty]$ such that $w(s)>s$ for all $s>0$. Also $w$ is semicontinuous from the right. We need only prove that

$$
w(d(f(x), f(y))) \leqq d(x, y), \quad x, y \in X .
$$

Suppose not. Then

$$
w(d(f(x), f(y)))>d(x, y)
$$

for some $x, y$ in $X$. Thus

$$
\epsilon \equiv d(f(x), f(y))>0 \text { and } d(x, y)<w(\epsilon) .
$$

By the choice of $w, d(f(x), f(y))<\epsilon$, a contradiction.

(ii) $\Rightarrow$ (i). Let $\epsilon>0$. Since $w$ is lower semicontinuous from the right at $\epsilon$, there exists $\delta_{1}(\epsilon)>0$ such that

$$
\frac{\epsilon+w(\epsilon)}{2}<w(s) \text { whenever } \epsilon \leqq s<\epsilon+\delta_{1}(\epsilon) \text {. }
$$

Let $\delta(\epsilon)=\min \left\{\delta_{1}(\epsilon),(w(\epsilon)-\epsilon) / 2\right\}$. Suppose that $\epsilon \leqq d(x, y)<$ $\epsilon+\delta(\epsilon)$. We need only to prove that $d(f(x), f(y))<\epsilon$. Suppose not. Then by the contractivity of $f$,

$$
\epsilon \leqq d(f(x), f(y))<\epsilon+\delta(\epsilon) \leqq \epsilon+\delta_{1}(\epsilon) .
$$




$$
\begin{aligned}
\frac{\epsilon+w(\epsilon)}{2} & <w(d(f(x), f(y))) \\
& \leqq d(x, y) \\
& \leqq \epsilon+\delta(\epsilon) \\
& \leqq \epsilon+\frac{w(\epsilon)-\epsilon}{2} \\
& =\frac{\epsilon+w(\epsilon)}{2}
\end{aligned}
$$

a contradiction.

As shown above, Theorem 1 gives Theorem A. Intuitively, one would think that the conditions on $f$ in Theorem B and (ii) of Theorem 1 should be equivalent. However, Theorem B is a special case of, and is not equivalent to Theorem A [3]. In other words, there is no symmetry in "right and left" in the sense that the fixed point theorems obtained depend on the sides - left or right - on which we impose monotone transformations. However, the following shows that such symmetry does exist if we restrict ourselves to the case where $w$ in (ii) of Theorem 1 is lower semicontinuous (or $\Phi$ in Theorem B is upper semicontinuous).

THEOREM 2. Let $f$ be a self map on a nonempty complete metric space $(X, d)$. Then the following conditions are equivalent:

(i) There exists a self map $\Phi$ on $[0, \infty)$ such that $\Phi(t)<t$ for $t>0, \Phi$ is increasing, continuous and $f$ is $\Phi$-contractive.

(ii) There exists a self map $w$ on $[0, \infty)$ such that $w(s)>s$ for $s>0, w$ is lower semicontinuous and

$$
w(d(f(x), f(y))) \leqq d(x, y), \quad x, y \in X
$$

For related fixed point theorems for function $f$ satisfying conditions in Theorem 2, we refer the reader to [2] and [5].

Added in proof: Indication of a proof for Theorem 2 is given in [6]: Chi Song Wong, Maps of Contractive Type, Proceedings of the Seminar on fixed point theory and its applications, Academic Press (1976), 197-207.

\section{REFERENCES}

1. D. W. Boyd and J. S. W. Wong, On nonlinear contractions, Proc. Amer. Math. Soc., 20 (1969), 458-464. 
2. F. E. Browder, On the convergence of successive approximations for nonlinear functional equations, Nederl Akad. Wetensch Proc., Ser. A71 - Indag. Math., 30 (1968), 27-35.

3. A. Meir and E. Keeler, A theorem on contraction mappings, J. Math. Anal. \& Appli., 28 (1969), 326-329.

4. E. Rakotch, A note on contraction mappings, Proc. Amer. Math. Soc., 13 (1962), 459-465.

5. Chi Song Wong, A fixed point theorem for a class of mappings, Math. Ann., 204 (1973), 97-103.

Received October 21, 1975 and in revised form July 16, 1976.

UNIVERSITY OF WINDSOR

Windsor, ONTARIO N9B 3P4, CANADA 



\section{Pacific Journal of Mathematics}

Vol. 68, No. 1

March, 1977

Richard Julian Bagby, On $L^{p}, L^{q}$ multipliers of Fourier transforms . .......

Robert Beauwens and Jean-Jacques Van Binnebeek, Convergence theorems in

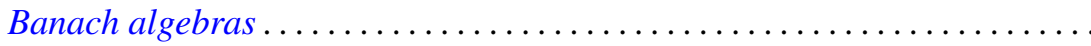

James Cyril Becker, Skew linear vector fields on spheres in the stable

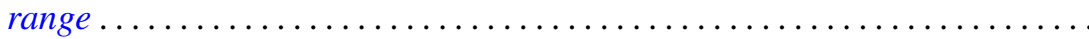

Michael James Beeson, Continuity and comprehension in intuitionistic formal

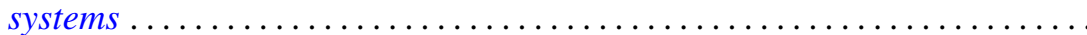

James K. Deveney, Generalized primitive elements for transcendental field

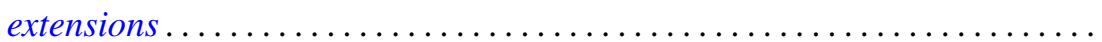

Samuel S. Feder, Samuel Carlos Gitler and K. Y. Lam, Composition properties

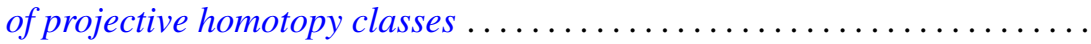

Nathan Jacob Fine, Tensor products of function rings under composition ......

Benno Fuchssteiner, Iterations and fixpoints . . . . . . . . . . . . . .

Wolfgang H. Heil, On punctured balls in manifolds

Shigeru Itoh, A random fixed point theorem for a multivalued contraction

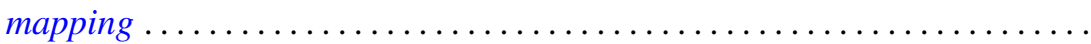

Nicolas P. Jewell, Continuity of module and higher derivations . . . . . . ......

Roger Dale Konyndyk, Residually central wreath products . . . . . . . . . . .

Linda M. Lesniak and John A. Roberts, On Ramsey theory and graphical

parameters.

Vo Thanh Liem, Some cellular subsets of the spheres.

Dieter Lutz, A perturbation theorem for spectral operators

P. H. Maserick, Moments of measures on convex bodies ... . . .

Stephen Joseph McAdam, Unmixed 2-dimensional local domains . .

D. B. McAlister and Norman R. Reilly, E-unitary covers for inverse semigroups...

William H. Meeks, III and Julie Patrusky, Representing codimension-one

homology classes by embedded submanifolds . . .

Premalata Mohapatro, Generalised quasi-Nörlund summability . .

Takahiko Nakazi, Superalgebras of weak-*Dirichlet algebras .

Catherine Louise Olsen, Norms of compact perturbations of operators .

William Henry Ruckle, Absolutely divergent series and isomorphism of

subspaces. II.

Bernard Russo, On the Hausdorff-Young theorem for integral operators .

Arthur Argyle Sagle and J. R. Schumi, Anti-commutative algebras and

homogeneous spaces with multiplications ............

Robert Evert Stong, Stiefel-Whitney classes of manifolds .

D. Suryanarayana, On a theorem of Apostol concerning Möbius functions of

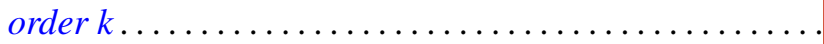

Yoshio Tanaka, On closedness of $C$ - and $C^{*}$-embeddings . . 